Research Paper

\title{
Up-regulated of Angiopoietin-Like Protein 4 Predicts Poor Prognosis in Cervical Cancer
}

\author{
Dan $\mathrm{Nie}^{1,2}$, Qianwen Zheng², Ling Liu², Xiguang $\mathrm{Mao}^{2}$, and Zhengyu Li ${ }^{1 凶}$ \\ 1. Department of Obstetrics and Gynecology, Key Laboratory of Birth Defects and Related Diseases of Women and Children, Ministry of Education, West \\ China Second University Hospital, Sichuan University, Chengdu,610041, People's Republic of China. \\ 2. Department of Gynecology, The Affiliated Hospital of Southwest Medical University, Luzhou, Sichuan 646000, People's Republic of China \\ $\square$ Corresponding author: Zhengyu Li, MD, West China Second University Hospital, Sichuan University, No. 20 Section 3, Renmin South Road, Chengdu, \\ Sichuan 610041, People's Republic of China. Tel: +86 18982151025; Fax: +86 (28) 85502391; Email: zhengyuli@scu.edu.cn \\ ( $)$ Ivyspring International Publisher. This is an open access article distributed under the terms of the Creative Commons Attribution (CC BY-NC) license \\ (https://creativecommons.org/licenses/by-nc/4.0/). See http://ivyspring.com/terms for full terms and conditions.
}

Received: 2018.09.13; Accepted: 2019.02.23; Published: 2019.04.21

\begin{abstract}
Background: Angiopoietin-like protein 4 (ANGPTL4) has been demonstrated highly expressed in some cancers, but it also was downregulated in others through DNA methylation. However, the expression status of ANGPTL4 in cancer of cervix remains unclear. Thus, our present study attempts to investigate the role of ANGPTL4 in the prognosis of patients with cervical cancer.

Methods: Immunohistochemistry, western blotting and QRT-PCR were performed to investigate the expressive level of ANPGTL4 in cervical cancer tissues and paired noncancer tissues. The relationship of ANGPTL4 expression and clinicopathological factors was measured by Chi-square test. Overall and disease-free survival (OS and DFS) rates were estimated and compared by using Kaplan-Meier method and log-rank test, respectively. To assess the prognostic significance of ANGPTL4 in cervical cancer patients, the Cox regression model was utilized to process univariate and multivariate analyses.

Results: ANGPTL4 was upregulated in cervical cancer samples and advanced tumor stage, deep stromal invasion, lymph node metastasis, lymphovascular space invasion, as well as poor OS and DFS were shown to be tightly correlated with it. Results of Cox multivariate analysis demonstrated that ANGPTL4 expression was an independent prognostic factor for OS and DFS in cervical cancer.

Conclusion: Upregulated ANGPTL4 seems to be a useful marker for poor prognosis in cervical cancer.
\end{abstract}

Key words: cervical cancer, angiopoietin-like protein 4, prognosis

\section{Introduction}

Cervical cancer is among the most common malignancy with the fourth highest incidence in global women. Annually, about 527,600 females are identified with cervical cancer and 265,700 are dead from this disease [1]. According to a recent statistic, approximately $85 \%$ of all the new cases of cervical cancer and $90 \%$ of all deaths occurred in the developing countries [1,2]. In China, the morbidity and mortality of cervical carcinoma were $7.5 / 100,000$ and 3.4/100 000, respectively and were demonstrated to be higher than commonly developed countries [3]. Although cervical cytology, human papilloma virus screening and early preventive intervention have been broadly used in our routine early monitoring and the strategies were proved to be efficient in reducing the morbidity and mortality [4]. Approximately $15-61 \%$ of patients who have developed early-stage disease may occur metastasis and 5-year survival rate of them was only 5\% [5]. Therefore, it is urgent to identify some novel biomarkers for detection of the patients with high risk of metastasis and recurrence.

ANGPTL4 (angiopoietin-like protein 4), a member of the angiopoietin-related family, was previously reported to play a crucial role in regulating angiogenesis and glucolipid metabolism [6,7]. Moreover, several previous studies have demonstrated ANGPTL4 exerted multiple even opposite effects in different types of cancerous tissues [8-14]. The upregulation of ANGPTL4 promoted 
tumor growth, progression, angiogenesis, invasion, metastasis and reduced overall survival [8-12]. ANGPTL4 was also indicated to influence tumor metastasis by targeting both blood vessels and corresponding tumor cells in cell lines of lung cancer and melanoma [12,13]. Moreover, in gastric cancer and urothelial carcinoma, the inhibited influence of ANGPTL4 on the tumor progression was considered as the result of epigenetic silencing by promoter methylation $[15,16]$. However, the clinical significance of ANGPTL4 in cervical cancer has not yet been well investigated.

In the current study, we first investigated the expression levels of ANGPTL4 in cervical cancer patients. Besides, the correlation between ANGPTL4 levels and clinicopathological characteristics or prognosis of patients with cervical cancer was also clarified.

\section{Materials and Methods}

\section{Patients and tissue specimens}

This study was approved by the Research Ethics Committee of The Affiliated Hospital of Southwest Medical University (approval no: 2016013). Prior written consent was obtained from all the patients.

160 cervical cancer specimens and matched noncancer specimens in the neighborhood were collected from patients (IA2-IIA stage) who underwent radical hysterectomy and lymphadenectomy from February 2009 to December 2011 in The Affiliated Hospital of Southwest Medical University. No patients had received chemotherapy or radiotherapy preoperatively. Diagnosis and grading of all cervical cancers were performed following the latest International Federation of Gynecology and Obstetrics (FIGO) guidelines [2]. The fresh paired specimens were transferred into liquid nitrogen until use and paraffin-embedded samples were also obtained from the department of pathology in our hospital.

\section{Follow-up}

All cervical cancer patients were followed-up in person as outpatients periodically. The follow-up time was initiated at the day of surgery and terminated in December 2016 (the study closing date) or death. The patients' ages ranged from 28-68 (median, 47) years old. The median follow-up duration was 59.3 (range, 5-94) months. Overall survival (OS) and Disease-free survival (DFS) were defined as previously described [14].

\section{RNA extraction and Quantitative real-time polymerase chain reaction ( $q R T-P C R$ )}

After extracting total RNA using Trizol
(Invitrogen, Carlsbad, CA, USA) and reverse transcription into cDNA by using RevertAid First Strand cDNA Synthesis Kit (Thermo, USA), Real-time PCR was performed using LightCycler ${ }^{\circledR} 480$ II Real-time PCR Instrument (Roche, Swiss). The specific primers were as follows: ANGPTL4 forward: $5^{\prime}$-AG ATGACCTCAGATGGAGGCTG-3'; and reverse, 5'CCAGCCAGAACTCGCCGT-3'; GAPDH forward:5'AGAAGGCTGGGGCTCATT-3'; and reverse: 5'-TGC TAAGCAGTTGGTGGTG-3'. The expression of mRNAs was normalized to GAPDH and was measured by the $2^{-\Delta \Delta C t}$ method [18].

\section{Immunohistochemistry}

Immunohistochemistry was carried out according to the protocols described in the previous study [19]. The sections were incubated overnight with the anti-ANGPTL4 antibody (1:100; Abcam, Cambridge, MA, USA) at $4^{\circ} \mathrm{C}$. At last, a secondary rabbit anti-goat immunoglobulin antibody (ZSGB-Bio Ltd., Beijing, China) was added to the slides after PBS washing, and the reaction products were visualized by staining with diaminobenzidine (Dako Ltd., Glostrup, Denmark), followed by counterstaining with hematoxylin.

ANGPTL4 expression was quantified by using a system that considered both the ratio of positive cells and the staining intensity [17]. The marks for the ratio of positive cells were $0(\leq 5 \%), 1(6-25 \%), 2(26-50 \%)$, and $3(>50 \%)$. The marks for staining intensity were 0 (undetectable), 1 (weak), 2 (medium), and 3 (strong). The two marks were summed up to obtain the final marks. A final mark of 0-3 was regarded as low expression, whereas a final mark of $\geq 4$ was considered high expression.

\section{Western blotting}

The protein was loaded and separated using 10\% SDS-PAGE before transferred to Immobilon-P transfer membranes (PVDF). 5\% milk was used for blocking the membranes, which was subsequently incubated with primary antibodies against human-ANGPTL4 (1:1000, Abcam, Cambridge, MA, USA), GAPDH (1:5000, Abcam, Cambridge, MA, USA) overnight. Then wash in TBST and probe with the secondary antibody (anti-rabbit; Proteintech Group, Inc., China). The protein expressions were visualized by enhanced chemiluminescence.

\section{Statistical analysis}

All statistical analyses were calculated by GraphPad Prism ${ }^{\circledR} 7.00$ (GraphPad, La Jolla, Ca, USA). Associations of the clinicopathological characteristics and ANGPTL4 expression was assessed by using the chi-square test or Fisher's exact test. The Kaplan-Meier method and log-rank test were used to 
synthesize and compare survival curves according to ANGPTL4 expression. The significance of various parameters for survival was determined by the Cox proportional hazards model. A $P$ value $<0.05$ was treated as statistically significant; all $P$ values were two-sided.

\section{Results}

\section{ANGPTL4 expression in cervical cancer}

The clinicopathological information was obtained from the patients' medical records and was summarized in Table 1. The expression of ANGPTL4 mRNA was significantly higher in cervical cancer tissues than in noncancer cervical tissues $(P<0.001$; Figure 1A). The western blotting also demonstrated the upregulation of ANGPTL4 in cervical cancer tissues (Figure 1B). Moreover, immunohistochemical staining showed that ANGPTL4 was mainly expressed in the cytoplasm and few nuclei of positive cells of cervical cancer tissues, but not in the noncancer cervical tissues (Figure 1C).

\section{Uni- and Multi-variable analyses of clinicopathological factors associated with 5-year OS and DFS rates of cervical cancer patients}

Kaplan-Meier survival analysis and the log-rank test were used to determine the relationship between ANGPTL4 expression and survival in cervical cancer patients. The 5-year OS rates of high ANGPTL4 expression and low ANGPTL4 expression patients were $66.6 \%$ and $86.8 \%$, respectively. The 5-year DFS rate for high ANGPTL4 expression and low ANGPTL4 expression were $59.4 \%$ and $78.1 \%$, respectively. Thus, high expression of ANGPTL4 is seemed to be related to shorter OS $(P=0.002$; Figure $2 \mathrm{~A})$ and DFS $(P=0.007$; Figure $2 \mathrm{~B})$ in cervical cancer patients and the result was statistically significant.

\section{The association between ANGPTL4 expression and cervical cancer clinicopathological characteristics}

The 160 cervical cancer patients were divided into two groups according to the median expression level of ANGPTL4 mRNA. The correlation between ANGPTL4 expression and different clinicopathological factors of cervical cancer patients was shown in Table 2. We found ANGPTL4 expression was highly correlated with FIGO stage $(P=0.006)$, lymphovascular space invasion (LVSI, $P=0.039$ ), lymph node metastasis (LNM, $P=0.002)$ and depth of stromal invasion $(P=0.004)$, while it was not correlated to patient's age, tumor size, histologic grade, or parametrial extension $(P>0.05$; Table 2$)$.
Table 1. Clinicopathologic characteristics of patients with cervical cancer

\begin{tabular}{ll}
\hline Characteristics & Patients, $\mathbf{n}(\%)$ \\
\hline Age(years) & $79(49.4)$ \\
$\leq 47$ & $81(50.6)$ \\
$>47$ & \\
FIGO stage & $89(55.6)$ \\
IA2-IB2 & $71(44.4)$ \\
IIA & \\
Tumor size & $100(62.5)$ \\
$\leq 4 \mathrm{~cm}$ & $60(37.5)$ \\
$>4 \mathrm{~cm}$ & \\
Deep of invasion & $50(31.2)$ \\
$<1 / 2$ & $110(68.8)$ \\
$\geq 1 / 2$ & \\
LVSI & $26(16.2)$ \\
yes & $134(83.8)$ \\
no & \\
LNM & $49(30.6)$ \\
yes & $111(69.4)$ \\
no & \\
Parametrial infiltration & $39(24.4)$ \\
yes & $121(75.6)$ \\
no & \\
Histologic grade & $51(31.9)$ \\
Grade 1 & $92(57.5)$ \\
Grade 2 \\
Grade 3 & $17(10.6)$ \\
\hline
\end{tabular}

Abbreviations: FIGO: International Federation of Gynecology and Obstetrics;

LVIS: lymphovascular space invasion; LNM: lymph node metastasis.

Table 2. Association between ANGPTL4 expression and clinicopathological characteristics in cervical cancer

\begin{tabular}{|c|c|c|c|c|}
\hline \multirow[t]{2}{*}{ Variable } & \multirow{2}{*}{$\begin{array}{l}\text { Number of } \\
\text { Patients }(n=160)\end{array}$} & \multicolumn{2}{|l|}{ ANGPTL4 } & \multirow[t]{2}{*}{$P$ value } \\
\hline & & $\overline{\operatorname{Low}(n=65)}$ & $\operatorname{High}(\mathrm{n}=95)$ & \\
\hline Age(years) & & & & 0.119 \\
\hline$\leq 47$ & 79 & $26(39.5)$ & $53(60.5)$ & \\
\hline$>47$ & 81 & $39(52.1)$ & $42(47.9)$ & \\
\hline FIGO stage & & & & 0.006 \\
\hline IA2-IB2 & 70 & 37 (52.9) & $33(47.1)$ & \\
\hline IIA & 90 & $28(31.1)$ & $62(68.9)$ & \\
\hline Tumor size & & & & 0.25 \\
\hline$\leq 4 \mathrm{~cm}$ & 110 & 48 (43.6) & $62(56.4)$ & \\
\hline$>4 \mathrm{~cm}$ & 50 & $17(34.0)$ & $33(66.0)$ & \\
\hline Deep of invasion & & & & 0.004 \\
\hline$\geq 1 / 2$ & 80 & $23(28.8)$ & $57(71.2)$ & \\
\hline$<1 / 2$ & 80 & $42(52.5)$ & 38 (47.5) & \\
\hline LVIS & & & & 0.039 \\
\hline yes & 39 & $10(25.6)$ & $29(74.4)$ & \\
\hline no & 121 & 55 (45.5) & $66(54.5)$ & \\
\hline LNM & & & & 0.002 \\
\hline yes & 57 & $14(24.6)$ & $43(75.4)$ & \\
\hline no & 103 & 51 (49.5) & $52(50.5)$ & \\
\hline Parametrial extens & ion & & & 0.665 \\
\hline yes & 39 & 17 (43.6) & $22(56.4)$ & \\
\hline no & 121 & $48(39.7)$ & $73(60.3)$ & \\
\hline Histologic grade & & & & 0.894 \\
\hline Grade 1 & 51 & $21(41.2)$ & $30(58.8)$ & \\
\hline Grade 2 & 92 & $38(58.7)$ & $54(41.3)$ & \\
\hline Grade 3 & 17 & $6(64.7)$ & $11(35.3)$ & \\
\hline
\end{tabular}

In a univariate Cox regression analysis, the 5 -year OS rate was significantly correlated with ANGPTL4 expression $(P=0.004)$, FIGO stage $(P=$ 
$0.048)$ and LNM $(P<0.001)$ and the 5 -year DFS rate was significantly correlated with ANGPTL4 expression $(P=0.003)$, stromal invasion depth $(P=0.03)$, LVSI $(P=0.031)$ and LNM $(P=0.004$; Table 3$)$. In a multivariate Cox proportional hazards model analysis, upregulation of ANGPTL4 $(P=0.038$, hazard ratio $[\mathrm{HR}]=2.191,95 \%$ confidence interval $[\mathrm{CI}]=1.046-$ 4.592; $P=0.027, \mathrm{HR}=3.66,95 \% \mathrm{CI}=1.160-11.544$, respectively) and lymph node metastasis $(P=0.011$, $\mathrm{HR}=2.931,95 \% \mathrm{CI}=1.284-6.691 ; P=0.025, \mathrm{HR}=$ $3.509,95 \% \mathrm{CI}=1.175-10.482$, respectively) were demonstrated as independent predictors for poor OS and DFS (Table 4).

\section{Discussion}

ANGPTL4 is a secreted glycoprotein that regulates lipoprotein metabolism, glucose homeostasis, angiogenesis, and wound healing [21-23]. Multiple studies revealed that whether ANGPTL4 behaved as a tumor-suppressor gene or an oncogene depended on the types of cancerous tissues [22]. By binding to integrins, ANGPTL4 promotes NADPH oxidasedependent $\mathrm{O}_{2}{ }^{-}$production to accelerates tumor growth, which in turn activates phosphoinositide 3kinase/protein kinase $\mathrm{Ba}$ and extracellular signalregulated kinase (ERK) pathways in an Src-dependent manner [24]. When overexpressed, it promotes neoplasm metastasis via the VCAM-1/integrin- $\beta 1$ signaling pathway and upregulates metalloproteinases, bone morphogenetic protein 7 , and fibronectin [9,25-27]. Moreover, its downregulation inhibits epithelial-mesenchymal transition by suppressing ERK signaling and, consequently, suppresses the proliferation, migration, and invasion of lung tumor

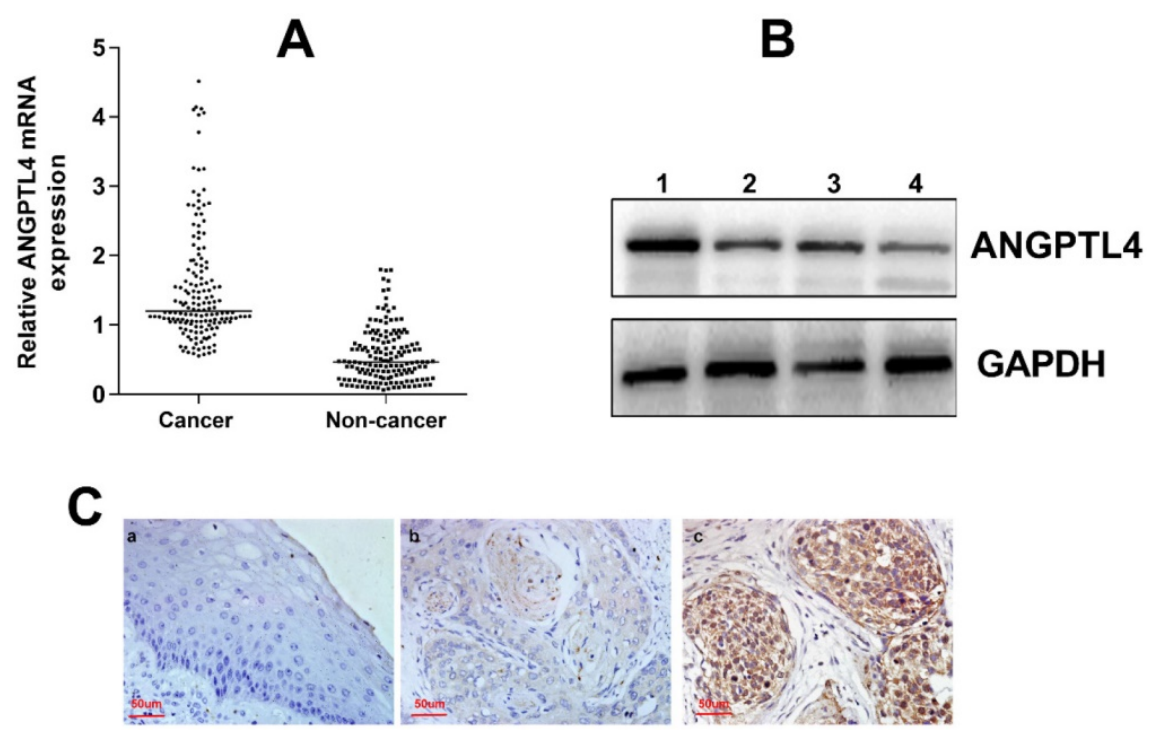

Figure 1. A. AGNPTL4 mRNA expression in cervical cancer tissues (cancer) and paired noncancer cervical tissues (non-cancer). $P<0.001$. B. The expression of ANGPTL4 detected by western blotting (1,3, cervical cancer tissues; 2,4, noncancer cervical tissues). C. Immunohistochemical staining of ANGPTL4 expression. a, Low ANGPTL4 expression in noncancer cervical tissue. b, Low ANGPTL4 expression in cervical cancer tissue. c, High ANGPTL4 expression in cervical cancer tissue.

Magnification, $\times 400$. cells [12]. In another hand, $\mathrm{Ng}$ et al. found fewer copies of the gene encoding ANGPTL4 was expressed in hepatocellular carcinoma tissues compared with non-tumor tissues and the tumor growth, angiogenesis, and metastasis were also suppressed when ANGPTL4 was upregulated [14]. Furthermore, methylation silencing of ANGPTL4 may promote tumor development [28]. Another study by Okochi-Takada et al. also showed that ANGPTL4 acted as a tumor suppressor and inhibited angiogenesis partly by inhibiting ERK signaling [15]. However, no reports have investigated the prognostic role of ANGPTL4 in cervical cancer.

Table 3. Univariate Cox analysis of OS and DFS in cervical cancer patients

\begin{tabular}{|c|c|c|c|c|}
\hline \multirow[t]{2}{*}{ Variable } & \multicolumn{2}{|l|}{ OS } & \multicolumn{2}{|l|}{ DFS } \\
\hline & HR (95\% CI) & $P$ value & HR $(95 \%$ CI) & $P$ value \\
\hline FIGO stage & & 0.048 & & 0.086 \\
\hline IA2-IB2 & 1 & & 1 & \\
\hline IIA & $2.024(1.006-4.068)$ & & $2.392(0.884-6.47)$ & \\
\hline Deep of invasion & & 0.641 & & 0.03 \\
\hline$<1 / 2$ & 1 & & 1 & \\
\hline$\geq 1 / 2$ & $1.178(0.591-2.35)$ & & $3.988(1.146-13.88)$ & \\
\hline LVIS & & 0.186 & & 0.031 \\
\hline no & 1 & & 1 & \\
\hline yes & $1.713(0.772-3.802)$ & & 2.997 (1.106-8.12) & \\
\hline LNM & & $<0.001$ & & 0.004 \\
\hline no & 1 & & 1 & \\
\hline yes & $3.765(1.886-7.516)$ & & $4.091(1.555-10.767)$ & \\
\hline ANGPTL4 & & 0.004 & & 0.003 \\
\hline Low & 1 & & 1 & \\
\hline High & $2.874(1.413-5.844)$ & & $5.574(1.815-17.113)$ & \\
\hline
\end{tabular}

Abbreviations: OS: overall survival; DFS: disease-free survival; HR: hazard ratio; CI: confidence interval; FIGO: International Federation of Gynecology and

Obstetrics; LVIS: lymphovascular space invasion; LNM: lymph node metastasis. 
A

Overall Survival

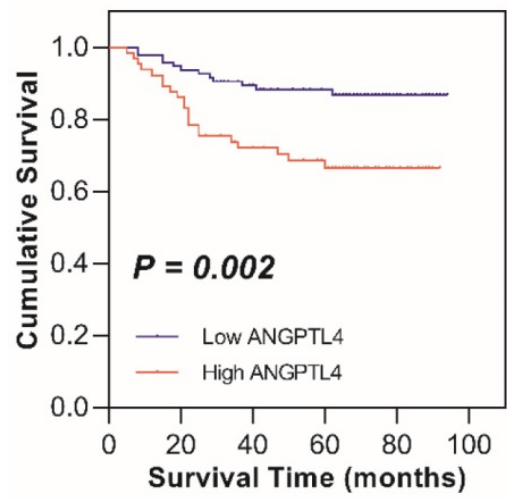

B

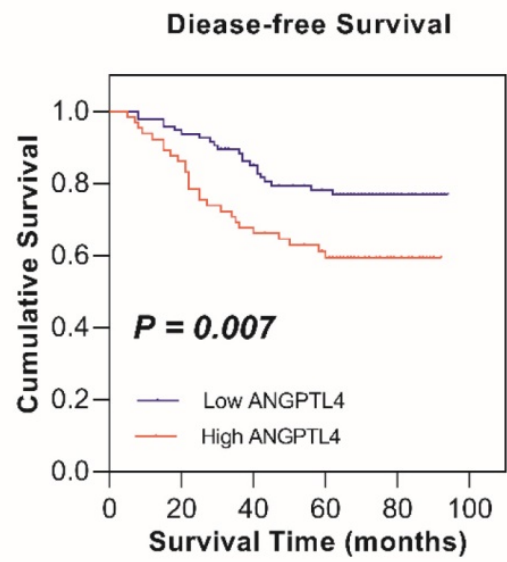

Figure 2. Kaplan-Meier survival analysis of low ANGPTL4 expression and high ANGPTL4 expression in patients with cervical cancer. A, Overall survival. B, Disease-free survival.

Table 4. Multivariate Cox regression analysis of OS and DFS in cervical cancer

\begin{tabular}{llllll}
\hline Variable & OS & & \multicolumn{1}{l}{ DFS } \\
\cline { 2 - 3 } \cline { 5 - 6 } & HR $(\mathbf{9 5 \%}$ CI) & P value & HR (95\% CI) & P value \\
\hline FIGO stage & $1.513(0.739-3.1)$ & 0.258 & & $1.619(0.593-4.421)$ & 0.348 \\
Deep of invasion & $0.807(0.396-1.646)$ & 0.555 & & $2.436(0.685-8.667)$ & 0.169 \\
LVIS & $1.229(0.537-2.81)$ & 0.625 & & $1.785(0.639-4.982)$ & 0.269 \\
LNM & $2.931(1.284-6.691)$ & 0.011 & & $3.509(1.175-10.482)$ & 0.025 \\
ANGPTL4 & $2.191(1.046-4.592)$ & 0.038 & & $3.66(1.16-11.544)$ & 0.027 \\
\hline
\end{tabular}

Abbreviation: OS: overall survival; DFS: disease-free survival; HR: hazard ratio; CI: confidence interval; FIGO: International Federation of Gynecology and

Obstetrics; LVIS: lymphovascular space invasion; LNM: lymph node metastasis.

Our findings demonstrated ANGPTL4 expression was upregulated in cervical cancer tissues than in surrounded noncancer tissues. According to previous reports, important prognostic factors in cervical carcinoma included tumor diameter, histological category, LNM, depth of stromal invasion, LVSI and parametrial extension [29]. In some previous studies, ANGPTL4 expression was pointed out to be related to tumor stage, LNM, vascular invasion, depth of stromal invasion, and reduced OS in various cancers $[8,10,11]$. In our study, upregulation of ANGPTL4 was also shown to be tightly related to tumor stage, depth of stromal invasion, LNM besides LVSI. However, no relationship between ANGPTL4 expression and patient's age, tumor size, parametrial extension, or histological grade was identified. Kaplan-Meier analysis results demonstrated that OS and DFS were significantly decreased in cervical cancer patients with upregulation of ANGPTL4. Moreover, COX univariate and multivariate proportional hazards regression analyses further confirmed that upregulation of ANGPTL4 might act as a biomarker of poor survival for cervical cancer patients.

However, there are still many limitations in the present study. First, the small sample size might reduce the statistical power of our results. In addition, although we have investigated the prognostic value of ANGPTL4 in cervical cancer patients, the potential tumorigenesis mechanism of ANGPTL4 in cervical cancer was not elucidated in the study.

In conclusion, our findings suggested that ANGPTL4 could be used as a marker of poor prognosis in cervical cancer patients. Otherwise, further investigation is needed to uncover the mechanism of the action of ANGPTL4 on cervical cancer.

\section{Acknowledgements}

Z.L. and D.N. conceived and designed this study. D.N., Q.Z., L.L., and X.M. collected and analyzed the data. D.N. and Q.Z. performed experiment and wrote the manuscript. All authors approved the final manuscript.

\section{Competing Interests}

The authors have declared that no competing interest exists.

\section{References}

1. Torre LA, Bray F, Siegel RL, Ferlay J, Lortet-Tieulent J, Jemal A. Global cancer statistics, 2012. CA Cancer J Clin. 2015; 65: 87-108.

2. Bermudez A, Bhatla N, Leung E. Cancer of the cervix uteri. Int J Gynaecol Obstet. 2015; 131 Suppl 2: S88-95.

3. Wang SM, Qiao YL. Implementation of cervical cancer screening and prevention in China--challenges and reality. Jpn J Clin Oncol. 2015; 45: 7-11.

4. Santesso N, Mustafa RA, Schünemann HJ, Arbyn M, Blumenthal PD, Cain J, et al. World Health Organization Guidelines for treatment of cervical intraepithelial neoplasia 2-3 and screen-and-treat strategies to prevent cervical cancer. Int J Gynaecol Obstet. 2016; 132: 252-8.

5. Pfaendler KS, Tewari KS. Changing paradigms in the systemic treatment of advanced cervical cancer. Am J Obstet Gynecol. 2016; 214: 22-30

6. Hato $\mathrm{T}$, Tabata $\mathrm{M}$, Oike $\mathrm{Y}$. The role of angiopoietin-like proteins in angiogenesis and metabolism. Trends Cardiovasc Med. 2008; 18: 6-14.

7. $\mathrm{Li} \mathrm{Y,} \mathrm{Teng} \mathrm{C.} \mathrm{Angiopoietin-like} \mathrm{proteins} \mathrm{3,} 4$ and 8: regulating lipid metabolism and providing new hope for metabolic syndrome. J Drug Target. 2014; 22: 679-87.

8. Shibata K, Nakayama T, Hirakawa $H$, Hidaka $S$, Nagayasu T. Clinicopathological significance of angiopoietin-like protein 4 expression in oesophageal squamous cell carcinoma. J Clin Pathol. 2010; 63: 1054-8. 
9. Li H, Ge C, Zhao F, Yan M, Hu C, Jia D, et al. Hypoxia-inducible factor 1 alpha-activated angiopoietin-like protein 4 contributes to tumor metastasis via vascular cell adhesion molecule-1/integrin $\beta 1$ signaling in human hepatocellular carcinoma. Hepatology. 2011; 54: 910-9.

10. Tanaka J, Irié T, Yamamoto G, Yasuhara R, Isobe T, Hokazono C, et al. ANGPTL4 regulates the metastatic potential of oral squamous cell carcinoma. J Oral Pathol Med. 2015; 44: 126-33.

11. Huang Z, Xie J, Lin S, Li S, Huang Z, Wang Y, et al. The downregulation of ANGPTL4 inhibits the migration and proliferation of tongue squamous cell carcinoma. Arch Oral Biol. 2016; 71: 144-9.

12. Zhu X, Guo X, Wu S, Wei L. ANGPTL4 Correlates with NSCLC Progression and Regulates Epithelial-Mesenchymal Transition via ERK Pathway. Lung. 2016; 194: 637-46.

13. Galaup A, Cazes A, Le JS, Philippe J, Connault E, Le CE, et al. Angiopoietin-like 4 prevents metastasis through inhibition of vascular permeability and tumor cell motility and invasiveness. Proc Natl Acad Sci U S A. 2006; 103: 18721-6.

14. Ng KT, Xu A, Cheng Q, Guo DY, Lim ZX, Sun CK, et al. Clinical relevance and therapeutic potential of angiopoietin-like protein 4 in hepatocellular carcinoma. Mol Cancer. 2014; 13: 196.

15. Okochi-Takada E, Hattori N, Tsukamoto T, Miyamoto K, Ando T, Ito S, et al. ANGPTL4 is a secreted tumor suppressor that inhibits angiogenesis. Oncogene. 2014; 33: 2273-8.

16. Hsieh HY, Jou YC, Tung CL, Tsai YS, Wang YH, Chi CL, et al. Epigenetic silencing of the dual-role signal mediator, ANGPTL4 in tumor tissues and its overexpression in the urothelial carcinoma microenvironment. Oncogene. 2018; 37: 673-86

17. Zhang M, Li N, Liang Y, Liu J, Zhou Y, Liu C. Hyaluronic acid binding protein 1 overexpression is an indicator for disease-free survival in cervical cancer. Int J Clin Oncol. 2017; 22: 347-52.

18. Kim SH, Park YY, Kim SW, Lee JS, Wang D, DuBois RN, ANGPTL4 induction by prostaglandin E2 under hypoxic conditions promotes colorectal cancer progression. Cancer Res. 2011; 71: 7010-20.

19. Livak KJ, Schmittgen TD. Analysis of relative gene expression data using real-time quantitative PCR and the 2(-Delta Delta C(T)) Method. Methods. 2001; 25: 402-8.

20. Huang Z, Bassil CF, Murphy SK. Methylation-specific PCR. Methods Mol Biol. 2013; 1049: 75-82.

21. Goh YY, Pal M, Chong HC, Zhu P, Tan MJ, Punugu L, et al. Angiopoietin-like 4 interacts with matrix proteins to modulate wound healing. J Biol Chem. 2010; 285: 32999-3009.

22. Tan MJ, Teo Z, Sng MK, Zhu P, Tan NS. Emerging roles of angiopoietin-like 4 in human cancer. Mol Cancer Res. 2012; 10: 677-88.

23. La Paglia L, Listì A, Caruso S, Amodeo V, Passiglia F, Bazan V, et al. Potential Role of ANGPTL4 in the Cross Talk between Metabolism and Cancer through PPAR Signaling Pathway. PPAR Res. 2017; 2017: 8187235.

24. Zhu P, Tan MJ, Huang RL, Tan CK, Chong HC, Pal M, et al. Angiopoietin-like 4 protein elevates the prosurvival intracellular $\mathrm{O} 2(-): \mathrm{H} 2 \mathrm{O} 2$ ratio and confers anoikis resistance to tumors. Cancer Cell. 2011; 19: 401-15.

25. Li X, Chen T, Shi Q, Li J, Cai S, Zhou P, et al. Angiopoietin-like 4 enhances metastasis and inhibits apoptosis via inducing bone morphogenetic protein 7 in colorectal cancer cells. Biochem Biophys Res Commun. 2015; 467: 128-34.

26. Liao $\mathrm{YH}$, Chiang KH, Shieh JM, Huang CR, Shen CJ, Huang WC, et al. Epidermal growth factor-induced ANGPTL4 enhances anoikis resistance and tumour metastasis in head and neck squamous cell carcinoma. Oncogene. 2017; 36: 2228-42.

27. Shen CJ, Chan SH, Lee CT, Huang WC, Tsai JP, Chen BK. Oleic acid-induced ANGPTL4 enhances head and neck squamous cell carcinoma anoikis resistance and metastasis via up-regulation of fibronectin. Cancer Lett. 2017; 386: $110-22$.

28. Hattori N, Okochi-Takada E, Kikuyama M, Wakabayashi M, Yamashita S, Ushijima T. Methylation silencing of angiopoietin-like 4 in rat and human mammary carcinomas. Cancer Sci. 2011; 102: 1337-43.

29. Biewenga P, van der Velden J, Mol BW, Stalpers LJ, Schilthuis MS, van der Steeg JW, et al. Prognostic model for survival in patients with early stage cervical cancer. Cancer. 2011; 117: 768-76. 